\title{
The Reviewing Process: Validating Results
}

By Ignacio Galiana and Pablo Cerrada

Any scientific publication aims to advance the field of knowledge that it deals with, and therefore the editorial staff will always be seeking the most revolutionary papers among all of those received. On the other hand, the reviewers' task is usually a much more conservative one, as reviewers are responsible for verifying the realism of the methods proposed and the veracity of the claimed results.

As the prestige of any scientific publication relies on both novelty and veracity of the papers published in it, validating the results presented in a paper is a major concern in the reviewing process, and it is crucial to the future of the publication. In this article, we focus on how to assess whether the results claimed by the authors are true, if they are exaggerated or fake, or if they have been copied from other works, i.e., plagiarism. Not appreciating the importance of this can lead to one of two extreme situations:

- an excellent article is rejected because its results are unbelievable to the reviewer

an article that exaggerates the results obtained, or in which these results are made up or copied from another author's work, is published.

Editors and reviewers should be aware of the former, as a rejected paper can be published in a competitor journal. A similar situation like this occurred when Enrico Fermi first submitted his work on the weak interaction theory of beta decay. Nature rejected the paper because it was considered unbelievable. Five years later, it was finally accepted when the results had already been proved [1].

The latter scenario usually has a very strong negative impact on the journal's prestige and credibility, and often ends in scandals when other researchers try to replicate the results claimed by the authors. Fortunately, these situations do not occur very often, but there are some recent examples that revealed some weak points of the editorial system. Kazuhiro Kosuge, past president of RAS, reported in the March 2011 issue of this magazine that, in 2010, serious plagiarism cases occurred and announced that the IEEE would take actions on the basis of IEEE ethics policies in the future.

Because they erode the credibility of the academic community, new methodologies should be implemented to avoid these issues. With some publications, authors can attach multimedia files to the submitted paper to support the presented results or methodologies. Although this can be a very helpful resource for evaluating the realism of a contribution, it is not very widely used; however, in some cases, it may be insufficient. During the reviewing process, the reviewer may encourage the authors to add a multimedia file to their submission to validate their results. 
Viewed this way, many researchers study both robotics and automation.

I should clarify the distinction between uppercase Quality and lowercase quality. Lowercase quality is related to value and, as Pirsig noted, is a subtle characteristic related to rigor and originality. A paper that emphasizes feasibility can be high quality and a paper emphasizing Quality can be of low quality.

Almost all papers include elements of both feasibility and Quality, the distinction will never be binary because it's a matter of degree. Both publications emphasize research over development. In my view, a paper with significant results that primarily emphasize feasibility (i.e., the focus is on proof of concept) should be submitted to $T-R O$. In contrast, a paper with significant results that primarily emphasize Quality (i.e., the focus is on performance) should be submitted to $T$-ASE. Of course, there will be many exceptions.

RAS is a successful marriage that has grown stronger over time. Now is a good time for the RAS community to take a fresh look at T-ASE and expand our definition of automation while also welcoming a new community of researchers who focus on automation. As I have argued here, an important part of this self-reflection is to expand and clarify our definition of automation.

Please consider submitting a paper; visit our Web site for updates, links to the latest papers, information about past and upcoming special issues (for example, on green manufacturing), a list of our editors and associate editors, and our new list of methodologies and applications. Also, please consider submitting to our Annual IEEE Conference on Automation Science and Engineering (IEEE-CASE).

I'm convinced that the RAS community will grow, thrive, and increase our global impact by advancing both robotics and automation. Maybe someone will even figure out a way for robots to maintain motorcycles.
This way of using already available tools for uploading multimedia files will be of great help when dealing with the situations described before: the reviewer will become convinced that the results are valid, and if the authors fail to upload the system working in these conditions, the reviewer will have evidence that suggests that the results are not as described in the paper.

Although using the available resources in such way would help improve the assessment of the results of a paper, the ideal way of evaluating whether an experiment is rigorous or not would be participating in a much more direct way on its design and then being present during its execution. Although this is not possible, we

Postscript: A version of this essay appeared in the January 2012 issue of T-ASE. T-ASE was astutely guided during its first three years by editorin-chief (EIC) Peter Luh and the next four years by EIC $\mathrm{N}$ Vishu Viswanadham. My term as EIC began in September 2011. I'm indebted to the entire T-ASE Editorial Board and the colleagues I consulted with on this essay, including Anto-

\section{A paper that} emphasizes feasibility can be high quality and a paper emphasizing Quality can be of low quality. nio Bicchi, Tim Bretl, Peter Corke, Alessandro De Luca, Seth Hutchinson, Vijay Kumar, Peter Luh, Kevin Lynch, Matt Mason, Bruno Siciliano, Frank van der Stappen, Dick Volz, and many others. Any blame for mistakes and omissions should be attributed to me. propose to implement a bidirectional communication channel among reviewers and authors to recreate the ideal scenario. Reviewers and authors should be able to dialogue, exchanging ideas and multimedia files. This bidirectional channel would allow the reviewers to anonymously participate under the supervision of the editor in the design of new experiments to backup the results of the paper, by asking the authors for graphic evidences of the results and sharing source codes.

Implementing such a tool is neither easy nor cheap, but we believe that increasing the confidence in publications and improving the certification of the published results is a capital matter for the future of scientific publications. Furthermore, these new methodologies will save huge amounts of time spent trying to replicate algorithms or methods that do not work as claimed, and finally will lead to a greater credibility of scientific publications.

Ignacio Galiana and Pablo Cerrada are part of SRP. For more information, visit the Web site at http://sites.ieee.org/ras-srp. They would like to thank Ludo Visser for his valuable suggestions about this article and his efforts with the SRP.

\section{Reference}

[1] (2011, Mar. 10). Notes on a scandal. Nature [Online]. 471, pp. 135-136. Available: http:// www.nature.com/nature/journal/v471/n7337/ full/471135b.html 